\title{
INDICADORES
}

\section{RESEARCHGATE COMO FUENTE DE EVALUACIÓN CIENTÍFICA: DESVELANDO SUS APLICACIONES BIBLIOMETRICAS}

\author{
ResearchGate as a source for scientific evaluation: \\ revealing its bibliometric applications
}

\section{Enrique Orduña-Malea, Alberto Martín-Martín y Emilio Delgado López-Cózar}

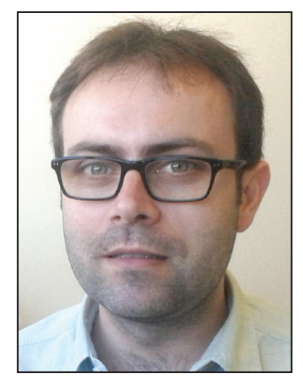

Enrique Orduña-Malea es doctor en documentación y premio extraordinario de tesis doctoral por la Universidad Politécnica de Valencia (UPV), en la que trabaja como investigador posdoctoral. Es miembro de los grupos de investigación Trademetrics (UPV) y EC3 Research Group (UGR). Sus principales líneas de investigación se centran en bibliometría y webmetría, especialmente en la elaboración de rankings, diseño y testeo de fuentes e indicadores web y su aplicación final a la evaluación de entornos académicos (principalmente autores y universidades) y comerciales (empresas y organizaciones).

http://orcid.org/0000-0002-1989-8477

Universidad Politécnica de Valencia, Instituto de Diseño y Fabricación (IDF) Camí de Vera, s/n. 46020 Valencia, España enorma@upv.es

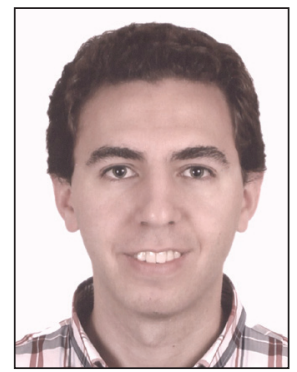

Alberto Martín-Martín, diplomado en biblioteconomía y documentación y licenciado en documentación por la Universidad de Granada $(U G R)$, recibió el Premio extraordinario de fin de carrera y el Premio nacional de fin de carrera en el área de ciencias sociales en 2013. Es miembro del grupo de investigación EC3 (UGR) y trabaja como personal de investigación FPU (formación de profesorado universitario) en esta misma universidad, donde elabora su tesis doctoral en el campo de la bibliometría y la comunicación científica. http://orcid.org/0000-0002-0360-186X

Universidad de Granada, Facultad de Comunicación y Documentación Campus de Cartuja, s/n. 18071 Granada, España albertomartin@ugr.es

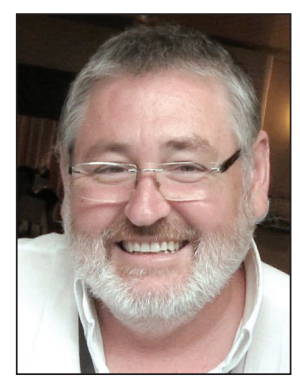

Emilio Delgado-López-Cózar es catedrático de metodología de investigación en la Universidad de Granada y fundador del grupo de investigación EC3 (Evaluación de la ciencia y de la comunicación científica). A lo largo de su trayectoria ha desarrollado un amplio catálogo de herramientas de evaluación científica, entre las que se incluyen In-RECS, In-RECJ e In-RECH (índices de impacto de las revistas españolas en ciencias sociales, jurídicas y humanidades), Índice h de las revistas científica españolas según Google Scholar Metrics, h-Index Scholar, Scholar Mirrors, Publishers' Scholar Metrics, Proceedings Scholar Metrics, Book Publishers Library Metrics, Co-Author Index, Classic Scholars' Profiles, Metaranking EC3 de universidades españolas, Ranking I-UGR de universidades españolas, RESH (Revistas españolas de ciencias sociales y humanidades), CIRC (Clasificación integrada de revistas científicas), Científica, y otros.

http://orcid.org/0000-0002-8184-551X

Universidad de Granada, Facultad de Comunicación y Documentación Campus de Cartuja, s/n. 18071 Granada, España edelgado@ugr.es

\section{Resumen}

ResearchGate es una de las más importantes redes sociales académicas, con más de 9 millones de usuarios y 80 millones de documentos. Además de importantes herramientas de networking social académico y ofertas de empleo, proporciona un amplio catálogo de indicadores bibliométricos, entre los que se encuentra ResearchGate Score. El objetivo principal de este trabajo es revelar las principales ventajas e inconvenientes de estos indicadores, prestando una atención especial al citado $R G$ Score, indicador insignia de ResearchGate. Pese a que ResearchGate ofrece unas prestaciones e indicadores bibliométri- 
cos con enormes posibilidades para obtener datos complementarios acerca del impacto de la producción científica y académica de un autor, tanto las políticas de comunicación como algunas acciones recientes tomadas en el diseño, elaboración y difusión de sus indicadores generan importantes dudas respecto a su uso con fines evaluativos. Finalmente, respecto a $R G$ Score, se concluye que el indicador no mide el prestigio de los investigadores sino su nivel de participación en la plataforma.

\section{Palabras clave}

ResearchGate; Redes sociales académicas; Indicadores bibliométricos; Evaluación científica.

\section{Abstract}

ResearchGate is currently one of the most important academic social networks, with more than 9 million users and 80 million documents. The platform offers not only important academic networking tools and job vacancies but also a wide range of bibliometric indicators. The objective of this work is to discuss the main advantages and disadvantages of these indicators paying special attention to the RG Score, the ResearchGate's flagship indicator. Although ResearchGate provides features and bibliometric indicators of enormous potential in order to gather additional insights about the scientific and academic impact of authors' production, their communication policies and some recent actions taken in the design, development, and dissemination of indicators generate serious doubts regarding their use for evaluation purposes. Finally, regarding the RG Score indicator, we conclude that it does not measure the prestige of researchers, but instead their level of participation in the platform.

\section{Keywords}

ResearchGate; Academic social networks; Bibliometric indicators; Altmetric indicators; Scientific evaluation.

Orduña-Malea, Enrique; Martín-Martín, Alberto; Delgado-López-Cózar, Emilio (2016). "ResearchGate como fuente de evaluación científica: desvelando sus aplicaciones bibliométricas". El profesional de la información, v. 25, n. 2, pp. 303-310.

http://dx.doi.org/10.3145/epi.2016.mar.18

\section{ResearchGate: una red social académica en} auge

ResearchGate es una red social académica fundada en Alemania en 2008 por dos médicos (Ijad Madish y Sören Hofmayer) y un informático (Horst Fickenscher), en la que trabajan actualmente más de 150 personas (cifra nada despreciable, si pensamos que el equipo de Google Scholar no supera la decena, por ejemplo).

El sistema se basa fundamentalmente en la capacidad para depositar y almacenar cualquier documento académico por parte de los autores (desde un artículo publicado en una revista de impacto hasta patentes, comunicaciones a congresos, materiales de un curso, una presentación o datasets) y en la inmediata obtención de estadísticas de uso personalizadas (quién visita, descarga o cita un documento o a sus autores).

Las prestaciones de red social vienen dadas principalmente por la capacidad y sencillez para interactuar con el resto de usuarios a través del seguimiento de actividades (followers y following) y de la participación directa mediante preguntas y respuestas. Finalmente, la plataforma proporciona un cada vez más potente servicio de búsqueda de empleo (principalmente contratos de investigación posdoctorales).

ResearchGate, que ya tenía en 2008 unos 10.000 usuarios, ha experimentado un crecimiento verdaderamente vertiginoso. Según la propia plataforma ${ }^{1}$, el 21 de octubre de 2015 había alcanzado los 9 millones de usuarios, aunque esta cifra es imposible de ser contrastada, pues no existe la forma de consultar el número real de perfiles públicos creados. No queda pues más remedio que confiar en las estadísticas de la empresa.
Durante sus largos 8 años de rodaje la plataforma ha ido evolucionando de forma ostensible (figura 1), a la par que crecían las inversiones millonarias que recibía, entre las que cabe destacar la del propio Bill Gates, quien donó la nada despreciable cifra de 35 millones de dólares a través de Tenaya Capital ${ }^{2}$, proporcionando de este modo un nuevo sentido a la desinencia del sistema: una verdadera "puerta" a los investigadores para difundir los resultados de sus investigaciones y contactar con otros colegas.

A fecha de febrero de 2016, la plataforma informa de la disponibilidad de más de 81 millones de publicaciones (de las que aproximadamente el $23,5 \%$ se encuentran a texto completo), con una representación de 193 países y con miembros verdaderamente insignes, entre los que se encuentran 52 investigadores galardonados con el premio $\mathrm{Nobel}^{3}$. De ser ciertos estos datos (la plataforma no permite obtener estos datos mediante una consulta) ResearchGate habría

Tabla 1. Tamaño de las principales bases de datos bibliográficas (marzo de 2016)

\begin{tabular}{|l|c|}
\hline \multicolumn{1}{|c|}{ Base de datos } & Número de documentos \\
\hline Google Scholar** & $170.000 .000^{*}$ \\
\hline Web of Science (todas las bases de datos) & 167.127 .889 \\
\hline ResearchGate & $\mathbf{8 1 . 0 0 0 . 0 0 0 *}$ \\
\hline Microsoft Academic Search & $80.000 .000^{*}$ \\
\hline Web of Science Core Collection & 61.856 .513 \\
\hline Scopus & 61.583 .942 \\
\hline Mendeley & $32.000 .000^{*}$ \\
\hline Academia.edu & $10.767 .769^{*}$ \\
\hline
\end{tabular}

* datos aproximados

** datos a fecha de junio de 2014 
superado en tamaño a Mendeley, Scopus y Web of Science Core Collection, convirtiéndose en una de las bases de datos bibliográficas más grandes del mundo, aunque todavía lejos de las cifras de Google Scholar (Orduña-Malea et al., 2015).

El éxito de esta red respecto a otras similares (como Academia.edu o los perfiles académicos de Mendeley) puede corroborarse de forma simple aunque contundente a través de Google trends (figura 2), así como a través de varios estudios y encuestas (Van Noorden, 2014; Kramer y Bosman, 2015) que la sitúan como una de las más empleadas por los científicos.

\section{ResearchGate como fuente de evaluación científica}

Como consecuencia tanto de su enorme popularidad como de los distintos indicadores que ofrece (no sólo a nivel individual sino de instituciones de investigación y empresas), el interés de la comunidad científica por ResearchGate no ha tardado en manifestarse. Así, estudios sobre la representatividad de los países y de las universidades (Thelwall; Kousha, 2015) o incluso de determinadas áreas y disciplinas académicas (González-Díaz; Iglesias-García; Codina, 2015) han aportado recientemente datos empíricos de gran interés acerca del potencial que supone la explotación de datos en ResearchGate. Además, una aproximación relacional basada en el análisis de redes sociales podría enriquecer la evaluación del impacto académico (Hoffman; Lutz; Meckel, 2016).

A esta notoriedad contribuye sin lugar a dudas la amplia y compleja red de métricas disponibles. En la tabla 2 se recogen los indicadores disponibles en la actualidad, organizados en función de la unidad de análisis (documento, autor, entidad, institución y cuestiones).

La disponibilidad gratuita de una batería de indicadores bibliométricos tan amplios y aparentemente sencillos facilita una inmediata caracterización de la reputación y estratificación científica de los autores. Esta circunstancia puede suponer una tentación para las instituciones, que pueden comenzar a utilizar estos valores de forma interna en procesos de evaluación de investigadores. ResearchGate incluye de hecho el $R G$ Score (su indicador insignia) en un lugar prominente del currículum académico que los autores pueden exportar automáticamente desde la plataforma. Por esta razón, tanto ésta en general como el $R G$ Score en particular, merecen una atención pormenorizada por parte de la comunidad bibliométrica.

\section{Algunas luces y demasiadas sombras}

El entramado de indicadores, unido al funcionamiento interno y la política de difusión de ResearchGate, nos proporciona algunas luces importantes de cara a la evaluación del rendimiento académico, aunque demasiadas sombras, que describimos a continuación.

\section{a) Luces en la evaluación: impacto de uso profesional}

Los indicadores de uso a nivel de documentos (principalmente el número de lecturas y descargas) ofrecen una dimensión complementaria y verdaderamente útil para conocer el impacto del material académico, especialmente de aquel no publicado en revistas de elite (o ni siquiera publicado), pero que es susceptible de lograr un destacado interés en determina- 
Tabla 2. Indicadores métricos disponibles en ResearchGate

\begin{tabular}{|l|c|c|c|c|c|}
\hline Métrica & Documento & Pregunta & Autor & Entidad & Institución \\
\hline Publications & -- & -- & $\mathrm{X}$ & $\mathrm{X}$ & $\mathrm{X}$ \\
\hline Reads & $\mathrm{X}$ & $\mathrm{No}$ & $\mathrm{X}$ & $\mathrm{X}$ & $\mathrm{X}$ \\
\hline Views & $\mathrm{X}$ (semanal) & $\mathrm{X}$ & $\mathrm{X}$ & No & No \\
\hline Citations & $\mathrm{X}$ & -- & $\mathrm{X}$ & No & No \\
\hline Citations (excluding self-citations) & $\mathrm{X}$ & -- & No & No & No \\
\hline Authors followed & -- & -- & $\mathrm{X}$ & No & No \\
\hline Authors following & -- & $\mathrm{X}$ & $\mathrm{X}$ & No & No \\
\hline Documents followed & -- & -- & $\mathrm{X}$ & No & No \\
\hline Documents following & -- & -- & No & No & No \\
\hline Questions following & -- & -- & $\mathrm{X}$ & No & No \\
\hline Questions asked & -- & -- & $\mathrm{X}$ & No & No \\
\hline Answers & -- & $\mathrm{X}$ & $\mathrm{No}$ & No & No \\
\hline Impact points & -- & -- & $\mathrm{X}$ & $\mathrm{X}$ & X \\
\hline Avg impact points & -- & -- & -- & $\mathrm{X}$ & No \\
\hline RG Score & -- & -- & $\mathrm{X}$ & No & X \\
\hline Members & -- & -- & -- & $\mathrm{X}$ & X \\
\hline h-index & -- & -- & $\mathrm{X}$ & No & No \\
\hline h-index (excluding self-citations) & -- & -- & $\mathrm{X}$ & No & No \\
\hline
\end{tabular}

X: métrica disponible; No: métrica no disponible; --: no procede.

das comunidades, especialmente las profesionales: aquellas que leen y usan pero no publican y por tanto no citan.

Aparte de conocer el número de lecturas, el sistema ofrece la oportunidad de conocer las personas específicas que están accediendo a un artículo (o cualquier otro material) prácticamente de forma instantánea, y desde qué instituciones ${ }^{4}$. Las oportunidades que esta opción brinda al networking académico y a la colaboración son inigualables.

RG Score no mide el prestigio de los investigadores, sino su grado de participación en la plataforma

Entre toda la producción depositada por un autor, la plataforma permite seleccionar (a diferencia de Google Scholar) aquella publicada en revistas, así como obtener el Factor de impacto (FI) de las revistas en las que el autor ha publicado, sin necesidad de acceder a los Journal Citation Reports (JCR).

El 8 de marzo de 2016, el equipo de ResearchGate presentaba como nueva métrica disponible el h-index ${ }^{5}$, tanto completo como excluyendo las autocitas. Este esfuerzo sitúa a la red académica en competencia directa con otras fuentes y plataformas (especialmente Google Scholar), que si bien ofrecen el h-index total, no permiten su cálculo eliminando las autocitas. Cabe recordar que, en la actualidad, solamente Scopus (de entre las principales bases de datos bibliográficas) permite el cálculo del h-index excluyendo las autocitas; la Web of science, aunque permite excluir las autocitas a la hora de calcular los datos totales de citas a un autor, no elimina éstas en el cómputo del h-index.

Estos indicadores, unidos a la creciente cobertura en número de documentos y número de usuarios en la plataforma, convierten a ResearchGate en una poderosa herramienta susceptible de ser usada con fines evaluativos. Por ejemplo, un reciente análisis aplicado a una muestra de 814 autores del campo de la bibliometría (Martín-Martín et al., 2016) muestra elevadas correlaciones (superiores a 0,8 ) entre los principales indicadores de Google Scholar (h-index y citas totales) y los principales indicadores de $R e$ searchGate (número de descargas, número de citas totales y $R G$ Score).

Estos resultados abren un importante camino para la obtención de evidencias de impacto académico en determinadas áreas y disciplinas científicas, a pesar de que existen todavía importantes limitaciones en el sistema automático de detección de referencias bibliográficas, que generan a su vez importantes errores en la asignación de citas a los autores.

\section{b) Sombras en la evaluación: opacidad, falta de trans- parencia, inestabilidad y manipulación}

No todo son luces en ResearchGate; y en concreto, su indicador más importante y representativo (RG Score) merece un análisis personalizado.

El objetivo de este indicador es ayudar a medir y calibrar la posición de un académico dentro de su comunidad científica, centrando para ello el interés en cómo la comunidad valora o recibe toda la investigación realizada por un autor, y

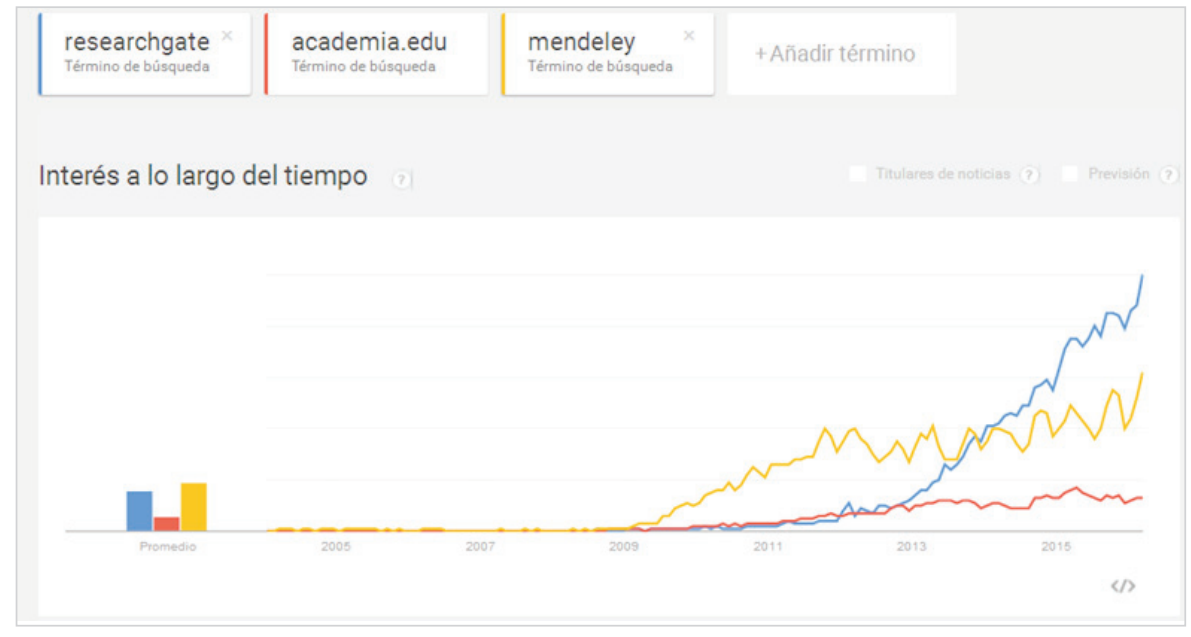

Figura 2. Evolución de la popularidad de las plataformas ResearchGate, Academia.edu y Mendeley (fuente: Google trends). 
no tanto por el material que éste ha publicado ${ }^{6}$. Para ello se parte de una radiografía de la actividad académica basada en tres dimensiones: las contribuciones (publicadas o no; sus citas, lecturas, descargas y, si procede, el Factor de impacto de la revista donde se publicó) que realiza un autor, su grado de interacción con la comunidad (alto o bajo; intervenir con preguntas y respuestas, seguir y ser seguido por otros usuarios, añadir comentarios y generar discusiones abiertas en los documentos a modo de open peer review, etc.) y su reputación (con quién interacciona, dónde publica, etc.).

El RG Score se desgrana en cuatro apartados: publicaciones, preguntas, respuestas, y seguidores. En cada uno de éstos contribuyen, de diferente forma, las distintas métricas ofrecidas en la tabla 1 . Por ejemplo, dentro del apartado "publicaciones" contribuye el número de documentos depositados, el número de lecturas, citas recibidas e Impact points. Sin embargo, el peso de cada métrica es desconocido.

Esta circunstancia ha hecho saltar todas las alarmas. En un estudio reciente $^{7}$, Peter Kraker, Katy Jordan y Elisabeth Lex trataron de reproducir el $R G$ Score y comprender así los distintos pesos de cada indicador. Lamentablemente, esta tarea fue imposible, aunque descubrieron que el indicador Impact points (sumatorio del Factor de impacto de las revistas en las que ha publicado un autor) tenía un peso destacado (en concreto, el logaritmo de este indicador). De ahí que pasar de un RG Score de 10 a 11 sea mucho más fácil que pasar de 20 a 21 puntos.

ResearchGate dispone de más de 81 millones de publicaciones, de las que aproximadamente el $23,5 \%$ se encuentran a texto completo

Más allá del uso de Impact points (cuya aplicación para la evaluación de personas debería ser rechazado de inmediato pues sumar factores de impacto de revistas de distinta naturaleza carece de sentido; no sólo no distingue entre disciplinas sino que utiliza indicadores aplicados a las revistas en lugar de medir el impacto de los documentos específicos de los autores), la elaboración, apertura y la transparencia de este indicador incumple todas las recomendaciones de buenas prácticas en la elaboración de indicadores bibliométricos del Manifiesto de Leiden (Hicks et al., 2015). Se puede destacar por ejemplo que diversos cambios internos en la metodología provocaron a finales de 2015 que los valores de $R G$ Score disminuyeran de un día para otro, cuando se trata de un indicador acumulativo; todo ello además sin la mediación de ninguna comunicación oficial. De este modo, ResearchGate reincide en las malas prácticas, tras la polémica fusión de views y downloads en un nuevo indicador, denominado Reads (MartínMartín et al., 2016).

\section{El RG Score merece una atención por- menorizada por parte de la comunidad bibliométrica}

La evolución de este indicador es constante. A principios de 2016 el $R G$ Score ofrecía, aparte del valor total acumulado, su variación semanal (figura 3 , arriba), en la que se podía comprobar visualmente el porcentaje que aportaba al valor final cada una de las dimensiones (publicaciones, preguntas, respuestas y seguidores). Sin embargo, desde principios de marzo de 2016 (junto con la aparición del h-index), la evolución semanal ha desaparecido (sin ofrecer ninguna explicación al respecto), resultando únicamente visible el valor $R G$ Score actual, añadiendo ahora la contribución de 

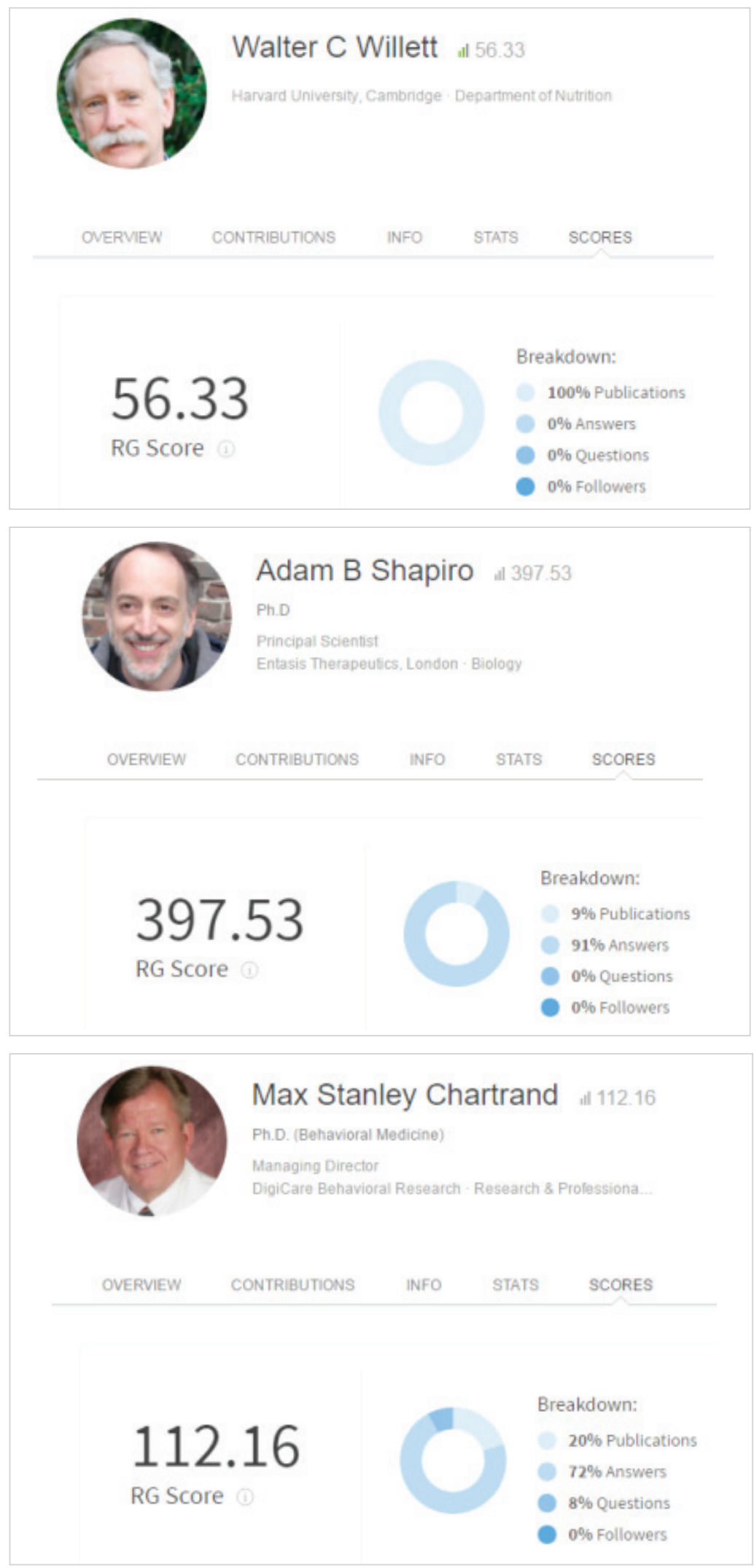

Figura 4. Perfiles ilustrativos del comportamiento empírico de RG Score

cada variable solamente al valor final (figura 3, abajo). Este hecho dificulta todavía más si cabe la disección e interpretación del indicador.

Dejando de lado el cuestionable diseño estadístico de $R G$ Score (de una mayor intervención mediante preguntas y res- puestas no se colige una mayor reputación científica), la opacidad de su cálculo, su falta de transparencia y su inestabilidad, el indicador es fácilmente manipulable.

Lo que supone una ventaja ideal (el depósito libre por parte de un autor de cualquier documento) se convierte en la práctica en una ausencia completa de control en la entrada de documentos al sistema: nada impide depositar una publicación falsa con la cantidad de autocitas que uno desee. A su vez, el número de Reads (el sumatorio de Summary reads, On-page reads, y downloads) pueden inflarse empleando "granjas" de autores. EI resultado directo es el crecimiento ilegítimo de citas recibidas, que puede repercutir directamente en el $R G$ Score.

Ahora bien, debemos advertir que el sistema ofrece dos controles importantes. En primer lugar, "todo lo que se hace, se ve". Las acciones de un determinado usuario (aunque algunas se pueden inhibir desde la zona de administración de la cuenta) dejan un rastro, por lo que las manipulaciones son fácilmente detectables por terceros. En segundo lugar, la inclusión del h-index excluyendo autocitas puede poner en evidencia ciertos currículums. A modo ilustrativo, el perfil público de Mostafa Eidiani ${ }^{8}$ muestra un hindex total de 65 , mientras que excluyendo las autocitas se queda en 11. En concreto, el sistema permite conocer fácilmente que el autor recibió durante marzo de 2016 un total de 2.785 citas, provenientes de un artículo y diversos documentos complementarios al mismo, formados éstos exclusivamente por referencias bibliográficas citando todas al autor, constituyendo un magnífico ejemplo de construcción de una granja de citas.

\section{RG Score: de lo oficial a lo oficioso}

Sin contar la total falta de transparencia o los cambios de política sin notificación alguna, los autores deseamos confrontar en este artículo lo que ResearchGate declara en su página oficial respecto al $R G$ Score y lo que sucede realmente.

Con ese fin, observemos algunos casos particularmente ilustrativos.

Declaración 1: "Como RG Score se basa en lo que tus colegas piensan de tu trabajo, nadie puede afectar directamente su propio $R G$ Score".

El perfil público del investigador Walter $\mathrm{C}$. Willett ${ }^{9}$ (figura 4; arriba), a fecha de 17 de marzo de 2016, presenta un $R G$ Score de 56,33 . El $100 \%$ de este indicador está construido a 
partir de los valores de la dimensión "publicación". Efectivamente, los números revelan un impacto científico de primer orden (189.190 citas; h-index de 226).

Sin embargo, analicemos igualmente el perfil correspondiente a Adam B. Shapiro ${ }^{10}$ (figura 4; medio), quien ostenta el valor más elevado de $R G$ Score localizado ( $R G=393,53$ ). Este perfil dispone de una cantidad importante de citas, pero sensiblemente inferior (2.860). En cambio, podemos observar cómo la dimensión "publicaciones" solamente contribuye un $9 \%$ a su RG Score, siendo la dimensión "Respuestas", con un $91 \%$, la que construye básicamente el valor de su indicador. Por tanto, un autor sí puede afectar directamente a su $R G$ Score sin necesidad de recurrir a la manipulación de citas, participando con preguntas y respuestas. La justificación viene dada porque ResearchGate considera una pregunta o respuesta como una contribución académica más:

"Una contribución es cualquier cosa que compartes en ResearchGate o añades a tu perfil. Tanto si se trata de preguntas y respuestas, como de un documento publicado que añades a tu perfil, unos resultados negativos o unos datos en bruto que cuelgues, todo lo que contribuyas puede contar para tu RG Score".

ResearchGate ofrece la oportunidad de conocer las personas específicas que están accediendo a un artículo prácticamente de forma instantánea

\section{Declaración 2: "Cuanto mayor sea la puntuación de los que interactúan con tu investigación, más aumen- tará tu propia puntuación"}

Un interesante ejemplo lo constituye el perfil de Max Stanley Chartrand ${ }^{11}$ (figura 4; abajo), quien posee un nada despreciable $R G$ Score de 112.16 pese a disponer únicamente de 41 citas y 23.54 Impact points. En cambio, la dimensión "Respuestas" contribuye al $72 \%$ de su RG Score, reforzando de este modo la evidencia de que la participación es la dimensión clave del indicador combinado.

En todo caso, todavía más curioso resulta observar que la dimensión "Seguidores" contribuye exactamente un $0 \%$, pese a disponer de 1.492 seguidores, algunos de ellos incluso con $R G$ Scores superiores a 100 . Por tanto, se demuestra empíricamente que la cantidad (y supuesta calidad) de los seguidores no influye en $R G$ Score.

\section{No todos los caminos llevan a mejorar el $R G$ Score}

Estos datos empíricos permiten caracterizar de una forma sencilla y contrastable el indicador RG Score:

- Depende de la actividad del científico en la plataforma: si éste sube material y, muy especialmente, si participa.

- No toda participación cuenta de la misma forma:

Responder: cuenta muchísimo.

Preguntar: cuenta mucho.

Publicar en revistas de alto impacto: cuenta bastante.
Ser leído (número de lecturas y descargas de documentos): cuenta poco.

Atraer la atención de los lectores (seguidores): no cuenta nada.

- No mide realmente la reputación. Únicamente aquellos $R G$ Scores construidos al $100 \%$ a través de la dimensión "publicaciones" son capaces de medir relativamente este aspecto.

- No valora realmente la interacción con otros científicos de prestigio.

Por tanto, se podría indicar que "todos los caminos llevan a $R G$ Score", pero unos caminos son más directos que otros. Las distintas rutas para alcanzar un valor alto son las siguientes:

- Participa en ResearchGate (preguntando y respondiendo, y recibiendo buenas valoraciones a tus respuestas), y lograrás incrementar rápidamente el $R G$ Score. Con este procedimiento llegarás al cielo.

- Publica en revistas de alto factor impacto, y mejorarás poco a poco tu RG Score. Con este procedimiento, no sólo habrás pasado por el purgatorio sino que te instalarás en él de manera sólida.

- Recibe muchas lecturas y descargas de documentos, y no te servirá prácticamente para nada. Con este procedimiento te mantendrás en la Tierra.

- Sigue a muchos científicos y atrae la atención de muchos otros (incluso de gran prestigio), y no te servirá de nada. Esta actividad te llevará al infierno (recibirás cientos de mensajes de la plataforma que te aturdirán si no desactivas inmediatamente las alertas).

La disponibilidad gratuita de una batería de indicadores bibliométricos tan amplios y aparentemente sencillos facilita una inmediata caracterización de la reputación científica de los autores

\section{Conclusiones}

La red social académica ResearchGate ofrece a los usuarios un amplio catálogo de indicadores tanto de impacto científico (citas) como de uso (lecturas, descargas) de documentos, así como una serie de indicadores combinados derivados de la actividad realizada por los usuarios en la plataforma.

Sin entrar a valorar las opciones de publicación o las distintas funcionalidades sociales de la plataforma, y centrando el análisis en los indicadores métricos disponibles exclusivamente, ResearchGate ofrece servicios y datos de inestimable valor para los investigadores: permite depositar y tipificar una amplia diversidad de materiales académicos (incluso registrar de forma gratuita DOls para éstos), conocer el interés del sector profesional y docente en ciertos materiales (presentaciones, cursos, etc.), conocer las personas e instituciones interesadas en tu trabajo, obtener datos de citas cada vez más completos según crece la cobertura de la plataforma (nada desdeñable en ciertas disciplinas como biomedicina), y fomentar la colaboración y networking académico. 
Sin embargo, pese a las evidentes ventajas y valiosas prestaciones de la plataforma, tanto las políticas de comunicación de ResearchGate como las acciones tomadas en el diseño, elaboración y difusión de diversos indicadores bibliométricos combinados (especialmente $R G$ Score), generan importantes dudas respecto a su uso con fines evaluativos.

$R G$ Score es un indicador insuficientemente explicado por parte de ResearchGate. Existe un alto grado de opacidad y falta de transparencia a la hora de describir los elementos que intervienen en su cálculo y el peso de cada uno de éstos. Pese a existir un razonable secreto comercial (ResearchGate es una empresa), la elaboración de indicadores bibliométricos que podrían ser usados en actividades de evaluación debería ser completamente transparente.

Además, se ha comprobado una falta de correspondencia entre algunas declaraciones oficiales acerca del funcionamiento del indicador y lo que empíricamente ofrece. Los resultados obtenidos muestran claramente que $R G$ Score es un indicador que mide fundamentalmente el grado de participación de un usuario en la plataforma ResearchGate y no el prestigio de un investigador, que es lo que la información oficial declara.

Existe un alto grado de opacidad y falta de transparencia a la hora de describir el funcionamiento y cálculo de $R G$ Score

Aparte de esta falta de correspondencia, $R G$ Score es un indicador inestable (los algoritmos de cálculo cambian constantemente sin ningún tipo de aviso) y no es posible replicarlo. Por tanto, el $R G$ Score es un pésimo indicador de rendimiento científico y debe ser desechado de forma enérgica por la comunidad científica, y no ser empleado en procesos de evaluación de la actividad científica.

En definitiva, concluimos que ResearchGate ofrece unas prestaciones y servicios con enormes posibilidades para obtener datos complementarios acerca del impacto del material académico, pero que manifiesta una serie de importantes desaciertos en sus políticas y en el diseño de sus indicadores, quedando por tanto un amplio espacio para la mejora.

\section{Notas}

\section{1. https://www.researchgate.net/press}

2. http://www.bloomberg.com/news/articles/2013-06-04/billgates-joins-35-million-investment-in-startup-researchgate

3. Los perfiles de los investigadores con este galardón aparecen con un membrete especial. Por ejemplo:

https://www.researchgate.net/profile/Paul_Modrich

4. Esta opción puede ser desactivada por aquellos usuarios que no deseen que otros autores sepan que han accedido a su material, por cuestiones de privacidad.

5. https://www.researchgate.net/blog/post/introducingthe-h-index-on-researchgate
6. $R G$ Score.

https://www.researchgate.net/publicprofile.RGScoreFAQ.htmI

7.http://blogs.Ise.ac.uk/impactofsocialsciences/2015/12/09/ the-researchgate-score-a-good-example-of-a-bad-metric

8. https://www.researchgate.net/profile/Mostafa_Eidiani/ stats/citations

9. https://www.researchgate.net/profile/Walter_Willett

10. https://www.researchgate.net/profile/Adam_Shapiro

11. https://www.researchgate.net/profile/Max_Chartrand

\section{Bibliografía}

González-Díaz, Cristina; Iglesias-García, Mar; Codina, Lluís (2015). "Presencia de las universidades españolas en las redes sociales digitales científicas: caso de los estudios de comunicación". El profesional de la información, v. 24, n. 5, pp. 1699-2407.

http://dx.doi.org/10.3145/epi.2015.sep.12

Hicks, Diana; Wouters, Paul; Waltman, Ludo; De-Rijcke, Sarah; Rafols, Ismael (2015). "Bibliometrics: The Leiden manifesto for research metrics". Nature, v. 520, n. 7548, pp. 429-431. http://dx.doi.org/10.1038/520429a

Hoffmann, Christian-Pieter; Lutz, Christoph; Meckel, Miriam (2016). "A relational altmetric? Network centrality on ResearchGate as an indicator of scientific impact".Journal of the Association for Information Science and Technology, v. 67, n. 4 , pp. 765-775.

http://dx.doi.org/10.1002/asi.23423

Kramer, Bianca; Bosman, Jeroen (2015). "101 innovations in scholarly communication - the changing research workflow" [poster].

https://dx.doi.org/10.6084/m9.figshare.1286826.v1

Martín-Martín, Alberto; Orduña-Malea, Enrique; AyIlón, Juan M.; Delgado-López-Cózar, Emilio (2016). The counting house: measuring those who count. Presence of Bibliometrics, Scientometrics, Informetrics, Webometrics and Altmetrics in the Google Scholar Citations, ResearcherID, ResearchGate, Mendeley \& Twitter. EC3 Working Papers, 21.

http://arxiv.org/pdf/1602.02412

Orduña-Malea, Enrique; Ayllón, Juan M.; Martín-Martín, Alberto; Delgado-López-Cózar, Emilio (2015). "Methods for estimating the size of Google Scholar". Scientometrics, v. 104, n. 3, pp. 931-949.

http://dx.doi.org/10.1007/s11192-015-1614-6

Thelwall, Mike; Kousha, Kayvan (2015). "ResearchGate: Disseminating, communicating, and measuring scholarship?". Journal of the Association for Information Science and Technology, v. 66, n. 5, 876-889.

http://dx.doi.org/10.1002/asi.23236

Van-Noorden, Richard (2014). “Online collaboration: Scientists and the social network". Nature, v. 512, n. 7513, pp. 126-129. http://dx.doi.org/10.1038/512126a 